\title{
Magnetic and Resonance Properties of Ferrihydrite Nanoparticles Doped with Cobalt
}

\author{
S. V. Stolyar ${ }^{a, b *}$, R. N. Yaroslavtsev ${ }^{a}$, R. S. Iskhakov ${ }^{b}$ O. A. Bayukov ${ }^{b}$, D. A. Balaev ${ }^{a, b}$, \\ A. A. Dubrovskii ${ }^{b}$, A. A. Krasikov ${ }^{b}$, V. P. Ladygina ${ }^{c}$, A. M. Vorotynov ${ }^{b}$, and M. N. Volochaev \\ ${ }^{a}$ Siberian Federal University, Svobodny pr. 79, Krasnoyarsk, 660041 Russia \\ ${ }^{b}$ Kirensky Institute of Physics, Siberian Branch, Russian Academy of Sciences, \\ Akademgorodok 50/38, Krasnoyarsk, 660036 Russia \\ ${ }^{c}$ International Scientific Center for Organism Extreme States Research at the Presidium of the Krasnoyarsk Scientific Center, \\ Siberian Branch, Russian Academy of Sciences, \\ Akademgorodok 50, Krasnoyarsk, 660036 Russia \\ *e-mail: stol@iph.krasn.ru \\ Received August 10, 2016
}

\begin{abstract}
Powders of undoped ferrihydrite nanoparticles and ferrihydrite nanoparticles doped with cobalt in the ratio of $5: 1$ have been prepared by hydrolysis of $3 d$-metal salts. It has been shown using Mössbauer spectroscopy that cobalt is uniformly distributed over characteristic crystal-chemical positions of iron ions. The blocking temperatures of ferrihydrite nanoparticles have been determined. The nanoparticle sizes, magnetizations, surface anisotropy constants, and bulk anisotropy constants have been estimated. The doping of ferrihydrite nanoparticles with cobalt leads to a significant increase in the anisotropy constant of a nanoparticle and to the formation of surface rotational anisotropy with the surface anisotropy constant $K_{u}=1.6 \times$ $10^{-3} \mathrm{erg} / \mathrm{cm}^{2}$.
\end{abstract}

DOI: $10.1134 / \mathrm{S} 1063783417030301$

\section{INTRODUCTION}

At present, a lot of attention has been paid to dispersed magnetic nanoparticles based on iron [1]. Among iron oxyhydroxides, the greatest attention has been drawn to metastable ferrihydrite, the properties of which are determined by the composition, the structure, and the method used for the preparation of this compound [2]. The chemical formula of ferrihydrite is usually written in the following form: $5 \mathrm{Fe}_{2} \mathrm{O}_{3}$. $9 \mathrm{H}_{2} \mathrm{O}$. However, since ferrihydrite has a defect structure, the number of $\mathrm{OH}$ bonds can be changed. In ferrihydrite, there are two types of anion packings [3]. In the case of the formation of a ferrihydrite fragment with cubic packing, in which the anion planes are arranged in the sequence $A B C A B C$, the $\mathrm{Fe}^{3+}$ ions are located in octahedral sites, thus forming two adjacent layers of octahedra occupied by iron atoms. At the same time, the formation of a ferrihydrite fragment with hexagonal packing, where the anion planes are arranged in the sequence $A B A B(A C A C)$, leads to the formation of single layers of octahedra [4]. Ferrihydrite is formed in the core of a protein complex, namely, ferritin, which represents a protein cage of apoferritin (the outer and inner diameters are equal to 12 and 5-8 nm, respectively) [5]. This complex exists in organs of almost all higher animals and fulfills the function of iron storage ("depot").

Massive ferrihydrite is an antiferromagnetic (AF) material. However, with a decrease in the size of antiferromagnetic particles to the nanometer scale, the magnetic properties of the material radically change. First, a decrease in the size of antiferromagnetic particles leads to an increase in the antiferromagnetic susceptibility, as well as to a change of its temperature dependence $[6,7]$. Second, the field of the spin-flop transition becomes weaker and the Néel temperature of ferrihydrite particles decreases [8, 9]. Third (and this is a qualitatively new effect), the ferrihydrite nanoparticles acquire a constant magnetic moment, which is caused by the incomplete compensation of the magnetic moments of the sublattices in such small objects. As a result, from the magnetic point of view, each ferrihydrite nanoparticle is an unusual "hybrid" of the antiferromagnet and the ferrite. Owing to the unusual properties acquired during the transition to a nanodispersed state, the ferrihydrite particles can compete with nanoparticles of conventional ferromagnetic and ferrimagnetic materials used in various practical applications [10], including those for the targeted delivery of drugs in an organism, as well as for the contrast in magnetic resonance imaging [11]. The field of 

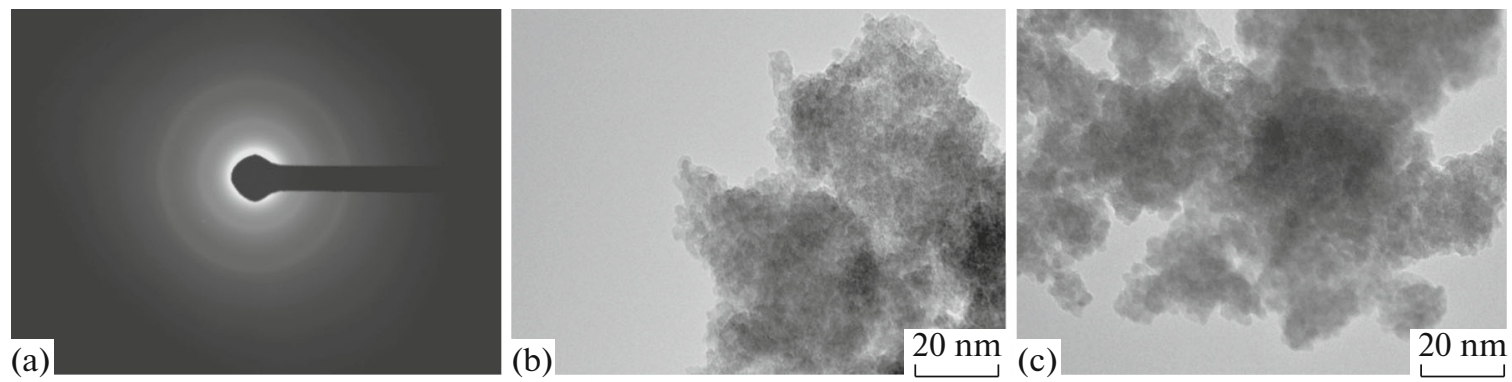

Fig. 1. (a) Microdiffraction pattern of the ferrihydrite nanoparticles and (b, c) transmission electron microscope images for (b) undoped ferrihydrite nanoparticles and (c) ferrihydrite nanoparticles doped with cobalt.

application of nanoparticles can be extended by means of the modification of their magnetic properties due to the doping [12]. In this study, we have investigated modifications of the static and dynamic magnetic properties of the chemically synthesized ferrihydrite nanoparticles and ferrihydrite nanoparticles doped with cobalt.

\section{SAMPLE PREPARATION AND EXPERIMENTAL TECHNIQUE}

Chemical ferrihydrite was synthesized by slow addition of an alkaline $\mathrm{NaOH}$ solution $(1 \mathrm{M})$ to a solution of ferric chloride $\mathrm{FeCl}_{3}(0.02 \mathrm{M})$ at room temperature with constant stirring until the $\mathrm{pH}$ reached a neutral value. The rate of addition of the alkali was varied in the range from 0.010 to $0.001 \mathrm{~mol} / \mathrm{min}$. The ferrihydrite sample doped with cobalt was synthesized in a similar way, but with the addition of the cobalt(III) salt to the reaction solution. The precipitate formed was collected on a filter. Then, the precipitate was washed and dried at room temperature.

The electron microscopy examination was carried out at the Center for Collective Use of the Krasnoyarsk Scientific Center of the Siberian Branch of the Russian Academy of Sciences (Krasnoyarsk, Russia) on a Hitachi HT7700 transmission electron microscope operating at an accelerating voltage of $100 \mathrm{kV}$. The Mössbauer spectra were measured on an MS-1104Em Mössbauer spectrometer with a ${ }^{57} \mathrm{Co}(\mathrm{Cr})$ source for powder samples with a thickness of 5$10 \mathrm{mg} / \mathrm{cm}^{2}$ according to the natural iron content. The low-temperature measurements were performed using a cryostat manufactured by OOO "KRIOTREID"
(Moscow, Russia). The chemical isomer shifts were given with respect to $\alpha$-Fe. The magnetic measurements were carried out on a vibrating-sample magnetometer [13]. The powder under investigation was fixed in a measuring cell filled with paraffin. The temperature dependences of the magnetic moment $M(T)$ were measured both in the mode of cooling without a magnetic field (zero field cooling (ZFC)) and in the mode of cooling in an external magnetic field (field cooling (FC)). The magnetic resonance spectra were recorded on a Bruker ELEXSYS 560 spectrometer operating in the $\mathrm{X}$ band mode (the characteristic microwave radiation frequency was $\sim 9.4 \mathrm{GHz}$ ) at temperatures in the range from 100 to $300 \mathrm{~K}$.

\section{RESULTS AND DISCUSSION}

\subsection{Results of the Transmission Electron Microscopy Investigations}

The results of the transmission electron microscopy investigations of the ferrihydrite nanoparticles prepared are presented in Fig. 1. Figure 1a shows the microdiffraction pattern, which is characteristic of ferrihydrite nanoparticles $[14,15]$. It can be seen from this figure that the microdiffraction pattern contains two diffuse reflections with the interplanar distances $d_{1}=1.6 \AA$ and $d_{2}=2.7 \AA$. The average size of the ferrihydrite nanoparticles is equal to $\sim 25 \AA$ (Fig. 1b). The doping with cobalt leads to an increase in the nanoparticle size to $35 \AA$ (Fig. 1c). Table 1 presents, in particular, the results obtained from the X-ray fluorescence analysis of the ferrihydrite nanoparticles doped with cobalt. According to the obtained results, the atomic concentrations of iron and cobalt are in the ratio $\mathrm{Fe}$ :

Table 1. Elemental composition of ferrihydrite nanoparticles

\begin{tabular}{l|c|c|c|c}
\hline \multirow{2}{*}{ Element } & \multicolumn{2}{|c|}{ Chemically synthesized ferrihydrite } & \multicolumn{2}{c}{ Ferrihydrite doped with cobalt } \\
\cline { 2 - 5 } & wt \% & at \% & wt \% & at \% \\
\hline $\mathrm{Fe}$ & 66.97 & 36.73 & 55.12 & 6.32 \\
$\mathrm{O}$ & 33.03 & 63.26 & 33.09 & 63.53 \\
$\mathrm{Co}$ & - & - & 11.79 & 6.15 \\
\hline
\end{tabular}




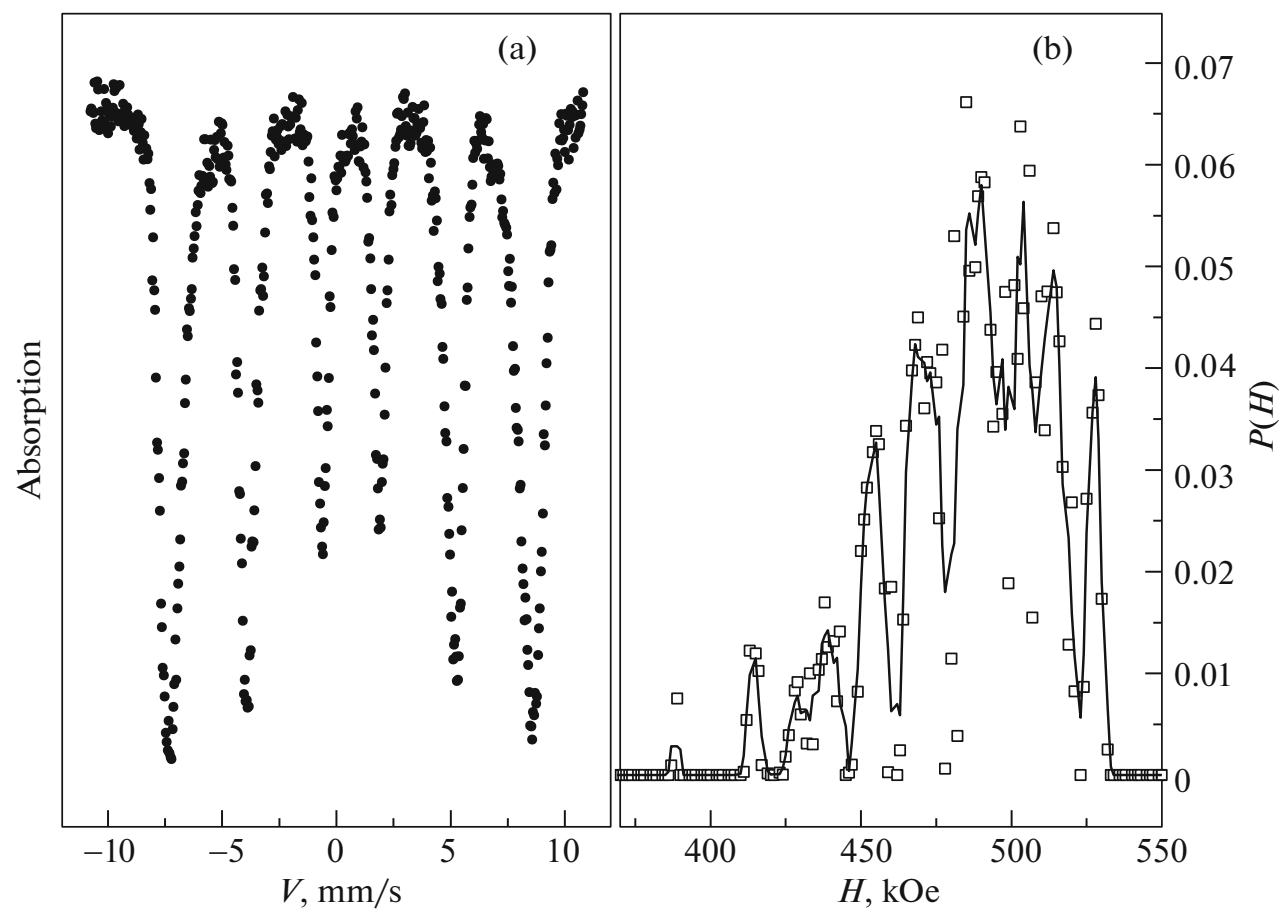

Fig. 2. (a) Mössbauer spectrum of the synthetic ferrihydrite measured at a temperature of $4 \mathrm{~K}$ and (b) probability distribution of the hyperfine fields in the experimental spectrum.

Co $=5: 1$. The microdiffraction patterns of the ferrihydrite nanoparticles doped with cobalt are identical to the microdiffraction pattern shown in Fig. 1a.

\subsection{Results of the Mössbauer Spectroscopy Investigations}

The Mössbauer spectrum measured for chemically synthesized ferrihydrite nanoparticles at a temperature of $4 \mathrm{~K}$ is shown in Fig. 2. This spectrum has the form of a Zeeman sextet. The interpretation of the spectrum was performed in two stages. At the first stage, we determined the distribution of hyperfine fields $P(H)$ in the experimental Mössbauer spectrum. Further, from the positions of the maxima, we estimated the number and parameters of nonequivalent positions occupied by the iron ions and then constructed a model Mössbauer spectrum. The model spectrum was fitted to the experimental spectrum by varying the entire set of hyperfine parameters. This fitting led to the vanishing of false spectral components and to the refinement of the parameters of the real subspectra. The parameters of the partial sextets, which were obtained from this interpretation, are presented in Table 2. The calculated parameters, in particular, the values of the hyperfine fields $H$, the occupancies of the iron positions $A$, and the quadrupole splittings QS, are in satisfactory agreement with the parameters of the Mössbauer spectra of ferrihydrites of natural and artificial origin [2]. The significant difference between the isomer shifts measured in two experiments can be associ- ated with different numbers of $\mathrm{OH}$ groups located in the environment of the $\mathrm{Fe}^{3+}$ ions, which substantially affects the electron density at the iron nucleus [4].

Figure 3 shows the Mössbauer spectra measured at room temperature for three ferrihydrite samples chemically synthesized at different rates of addition of the alkaline $\mathrm{NaOH}$ solution. These spectra have the form of quadrupole doublets, which are characteristic of unblocked superparamagnetic (SP) particles, with different degrees of line broadening. The analysis of the distribution of the quadrupole splittings $P(\mathrm{QS})$ in the experimental Mössbauer spectra (Fig. 3b) leads to the conclusion that, in the structure of the samples under investigation, there are at least three nonequiv-

Table 2. Mössbauer parameters

\begin{tabular}{c|c|c|c|c|c}
\hline $\begin{array}{c}\mathrm{IS}, \\
\mathrm{mm} / \mathrm{s}\end{array}$ & $H, \mathrm{kOe}$ & $\begin{array}{c}\mathrm{QS}, \\
\mathrm{mm} / \mathrm{s}\end{array}$ & $W, \mathrm{~mm} / \mathrm{s}$ & $A$ & Position \\
\hline 0.409 & 521 & 0 & 0.22 & 0.038 & - \\
0.639 & 516 & 0 & 0.29 & 0.070 & - \\
0.431 & 511 & 0 & 0.35 & 0.10 & - \\
0.492 & 491 & -0.03 & 0.56 & 0.473 & $\mathrm{Fe} 1$ \\
0.453 & 467 & 0 & 0.55 & 0.239 & $\mathrm{Fe} 2$ \\
0.464 & 436 & -0.01 & 0.56 & 0.080 & - \\
\hline
\end{tabular}

Here, IS is the chemical isomer shift with respect to $\alpha$-Fe, $H$ is the hyperfine field, QS is the quadrupole splitting, $W$ is the width of the absorption line, and $A$ is the area under the partial doublet (occupancy of the position). 

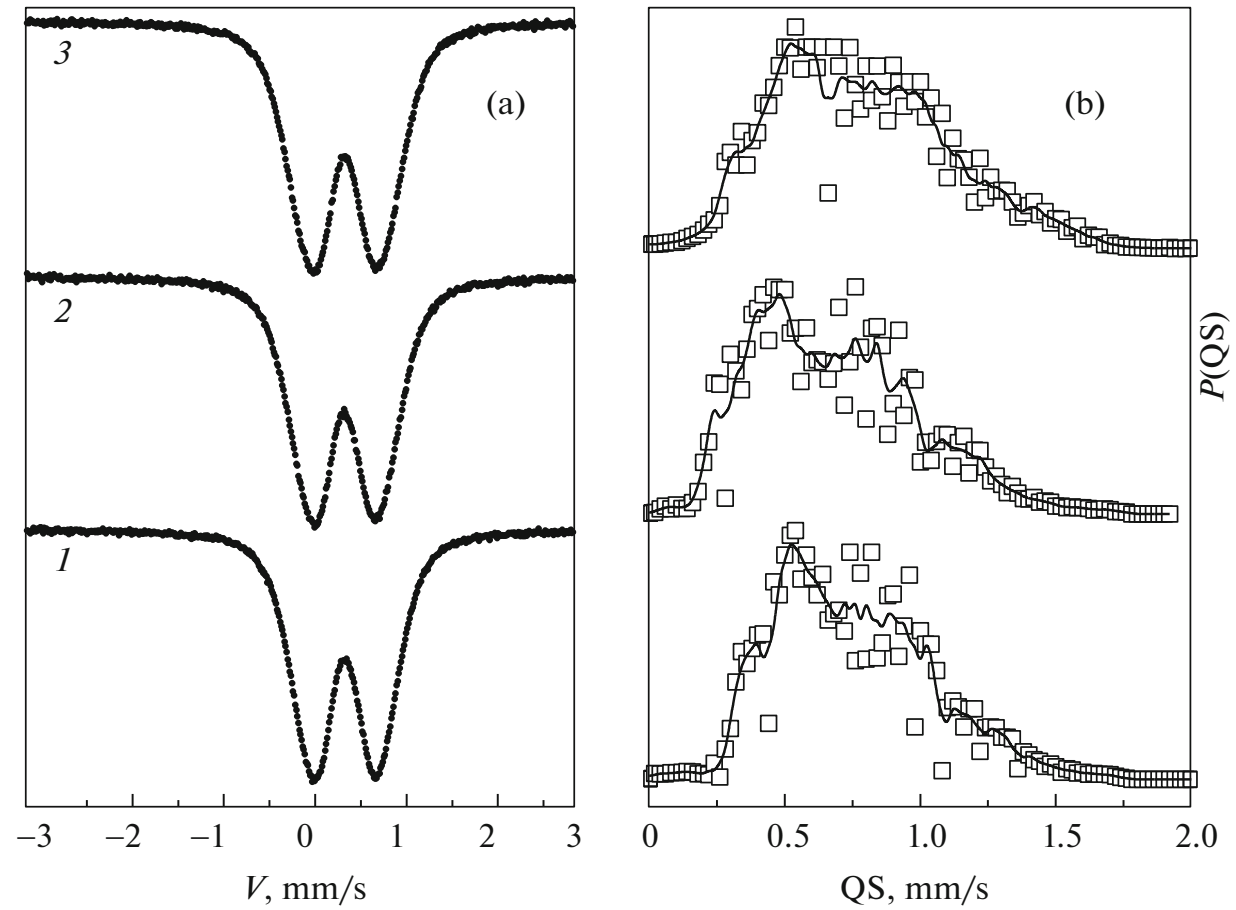

Fig. 3. (a) Mössbauer spectra of three chemically synthesized ferrihydrites and (b) probability distributions of the quadrupole splittings in the experimental spectra. The numbers of the spectra correspond to the sample numbers in Table 3 .

alent positions of iron with different degrees of distortion of the local environment. The model Mössbauer spectra were formed taking into account the specific features observed in the distribution $P(\mathrm{QS})$ and then were fitted to the experimental spectra by varying the entire set of hyperfine parameters. The results of the interpretation of the Mössbauer spectra of the chemically synthesized ferrihydrites are summarized in Table 3. The iron positions designated as $\mathrm{Fe} 1$ and $\mathrm{Fe} 2$ correspond to the cubic $(A B C A B C)$ and hexagonal

Table 3. Mössbauer parameters of the chemically synthesized ferrihydrites

\begin{tabular}{c|c|c|c|c|c}
\hline $\begin{array}{c}\text { Sample } \\
\text { no. }\end{array}$ & IS, mm/s & $\begin{array}{c}\mathrm{QS}, \\
\mathrm{mm} / \mathrm{s}\end{array}$ & $W, \mathrm{~mm} / \mathrm{s}$ & $A$ & Position \\
\hline 1 & 0.348 & 0.50 & 0.35 & 0.434 & $\mathrm{Fe} 1$ \\
& 0.351 & 0.81 & 0.33 & 0.371 & $\mathrm{Fe} 2$ \\
& 0.350 & 1.15 & 0.36 & 0.196 & $\mathrm{Fe} 3$ \\
2 & 0.344 & 0.50 & 0.37 & 0.453 & $\mathrm{Fe} 1$ \\
& 0.349 & 0.83 & 0.34 & 0.368 & $\mathrm{Fe} 2$ \\
& 0.347 & 1.19 & 0.36 & 0.179 & $\mathrm{Fe} 3$ \\
3 & 0.348 & 0.49 & 0.36 & 0.401 & $\mathrm{Fe} 1$ \\
& 0.352 & 0.83 & 0.35 & 0.365 & $\mathrm{Fe} 2$ \\
& 0.350 & 1.19 & 0.38 & 0.233 & $\mathrm{Fe} 3$ \\
\hline
\end{tabular}

$(A B A B)$ packings of the ligands, respectively, whereas the $\mathrm{Fe} 3$ positions correspond to interlayer iron ions.

The Mössbauer spectrum and the distribution of quadrupole splittings $P(\mathrm{QS})$ of the ferrihydrite nanoparticles doped with cobalt are shown in Fig. 4. This spectrum was measured at room temperature. The results of the interpretation of the Mössbauer spectrum of the ferrihydrite nanoparticles doped with cobalt are presented in Table 4. It can be seen from this table that, upon doping of the ferrihydrite nanoparticles with cobalt, the parameters of the Mössbauer spectra remain unchanged. The observed occupancies of the iron positions $\mathrm{Fe} 1, \mathrm{Fe} 2$, and $\mathrm{Fe} 3$ in cobaltdoped ferrihydrite nanoparticles are identical to the occupancies of these positions in the undoped ferrihydrite nanoparticles. The obtained results indicate that the cobalt atoms are uniformly distributed among the positions $\mathrm{Fe} 1, \mathrm{Fe} 2$, and $\mathrm{Fe} 3$.

Table 4. Mössbauer parameters of the ferrihydrite doped with cobalt

\begin{tabular}{c|c|c|c|c}
\hline IS, mm/s & QS, mm/s & $W, \mathrm{~mm} / \mathrm{s}$ & $A$ & Position \\
\hline 0.343 & 0.50 & 0.37 & 0.45 & $\mathrm{Fe} 1$ \\
0.350 & 0.82 & 0.33 & 0.34 & $\mathrm{Fe} 2$ \\
0.348 & 1.16 & 0.35 & 0.21 & $\mathrm{Fe} 3$ \\
0.351 & 1.48 & 0.26 & 0.04 & - \\
\hline
\end{tabular}




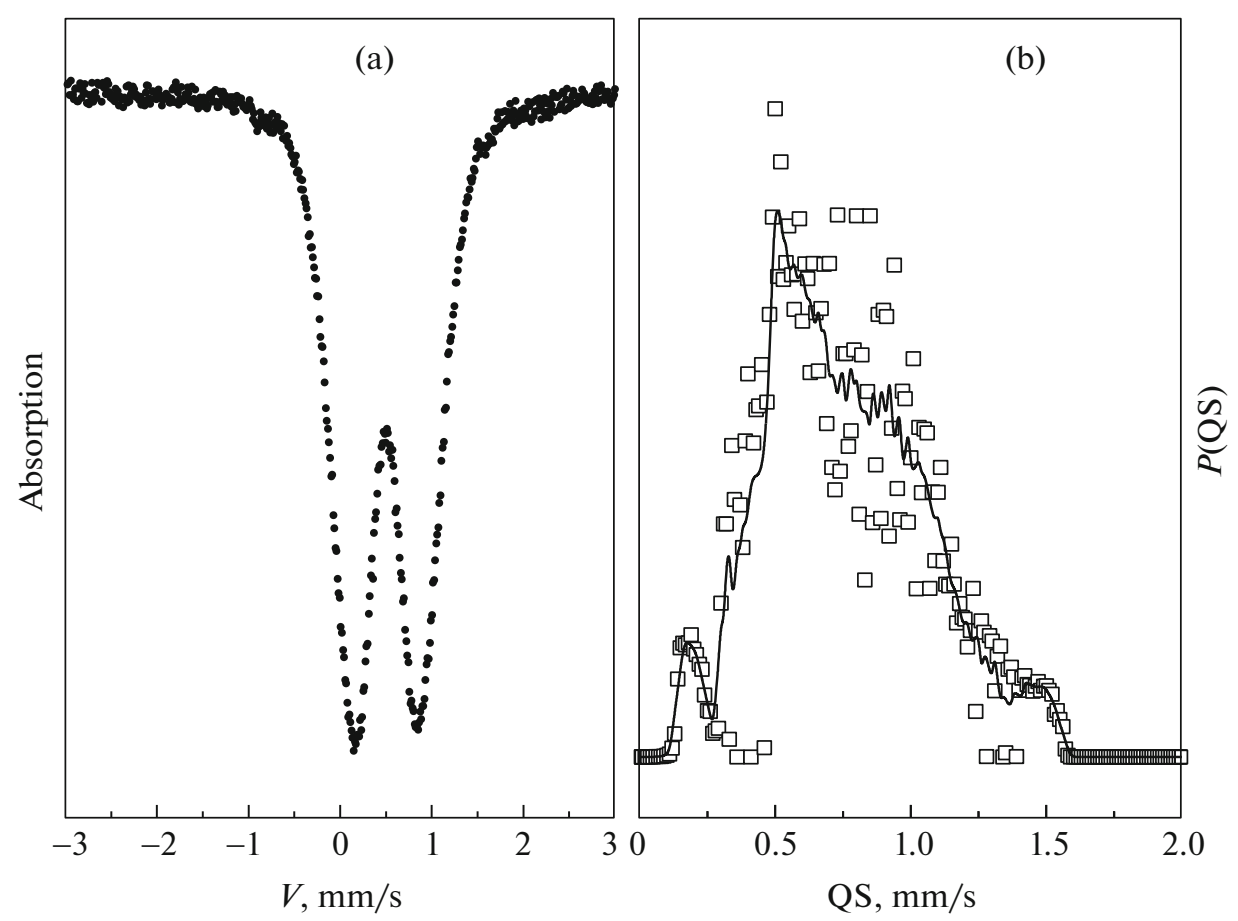

Fig. 4. (a) Mössbauer spectrum of the ferrihydrite nanoparticles doped with cobalt and (b) probability distribution of the quadrupole splitting in the experimental spectrum.

\subsection{Results Obtained on the Basis of the Temperature and Field Dependences of the Magnetization}

The temperature dependences $M(T)$ measured in the ZFC and FC modes (see insets in Figs. 5a and 5b) exhibit the characteristic superparamagnetic behavior. It can be seen from these figures that the dependences $M(T)_{\mathrm{ZFC}}$ have a maximum, in the vicinity of which there is a discrepancy with the dependence $M(T)_{\mathrm{FC}}$. In the temperature range below the blocking temperature $T_{\mathrm{B}}\left(T<T_{\mathrm{B}}\right)$, the dependences $M(H)$ demonstrate a hysteresis (Figs. 5a, 5b).

For a quantitative analysis of the dependences $M(H)$ at temperatures $T>T_{\mathrm{B}}$, we used an approach that is commonly accepted for systems of noninteracting antiferromagnetic nanoparticles, in which the magnetic moment of the sample is associated with the superparamagnetic behavior of individual particles with the inclusion of their distribution over the magnetic moments, as well as the component $\chi_{\mathrm{AF}} H$ of the magnetic susceptibility, which determines the antiferromagnetic contribution to the magnetization. In this case, the dependences $M(H)$ are described by the following expression [16-18]:

$$
\begin{gathered}
M(H)=N_{P} \\
\times \int^{\mu_{\max }} \mu_{\min } L\left(\mu_{P}, H\right) f\left(\mu_{P}\right) \mu_{P} d \mu_{P}+\chi_{\mathrm{AF}} H .
\end{gathered}
$$

In this expression, $L\left(\mu_{P}, H\right)=\operatorname{coth}\left(\mu_{P} H / k T\right)-$ $1 /\left(\mu_{P} H / k T\right)$ is the Langevin function, $f\left(\mu_{P}\right)$ is the dis- tribution function of the magnetic moment of the particles $\mu_{P}$, and $N_{P}$ is the number of particles per unit weight of the sample. We used the lognormal distribution $f\left(\mu_{P}\right)=\left(\mu_{P} s(2 \pi)^{1 / 2}\right)^{-1} \exp \left\{-\left[\ln \left(\mu_{P} / n\right)\right]^{2} / 2 s^{2}\right\}$, where $\left\langle\mu_{P}\right\rangle=n \exp \left(s^{2}\right)$ is the average value of the magnetic moment of the particle and $s^{2}$ is the variance of the quantity $\ln \mu_{P}$. During the processing of the obtained data in accordance with expression (1), we achieved the best agreement between the experimental and fitting curves [16] at each temperature. Using the temperature dependences $\left\langle\mu_{P}\right\rangle(T)$ and $\chi_{\mathrm{AF}}(T)$ with the extrapolation to the temperature $T=0 \mathrm{~K}$, we determined the values of the average magnetic moment $\mu_{P}(T)(T=0)$ and the antiferromagnetic susceptibility $\chi_{\mathrm{AF}}(T=0)$ (Table 5).

The values of the nanoparticle size $D$, the blocking temperature $T_{B}$, the coercive field $H_{C}$, the saturation magnetization $M_{S}$, and the antiferromagnetic susceptibility $\chi_{\mathrm{AF}}$ are presented in Table 5. For comparison, this table also presents the characteristics of ferrihydrite nanoparticles prepared by the cultivation of bacteria $[4,19,20]$.

\subsection{Results of the Ferromagnetic Resonance (FMR) Investigations}

The temperature dependences of the resonance fields and the ferromagnetic resonance linewidth depend on the chemical composition of the ferrihydrite nanoparticles under investigation. The ferromagnetic resonance curves of the ferrihydrite nanoparti- 
(a)

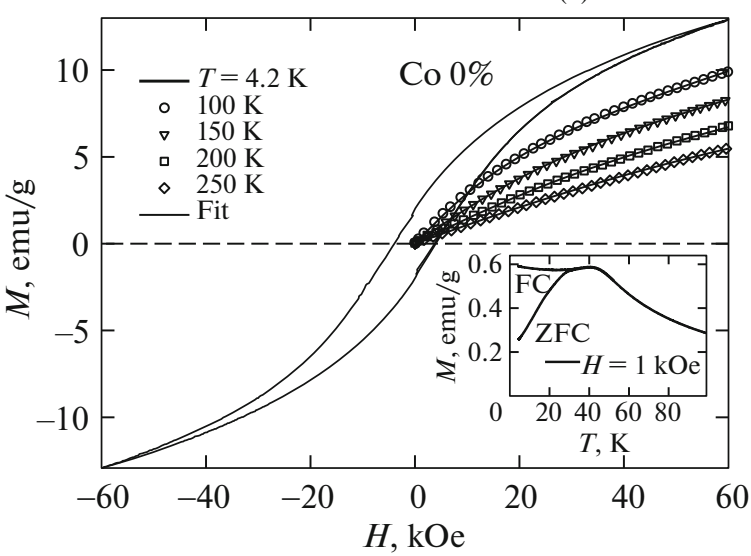

(b)

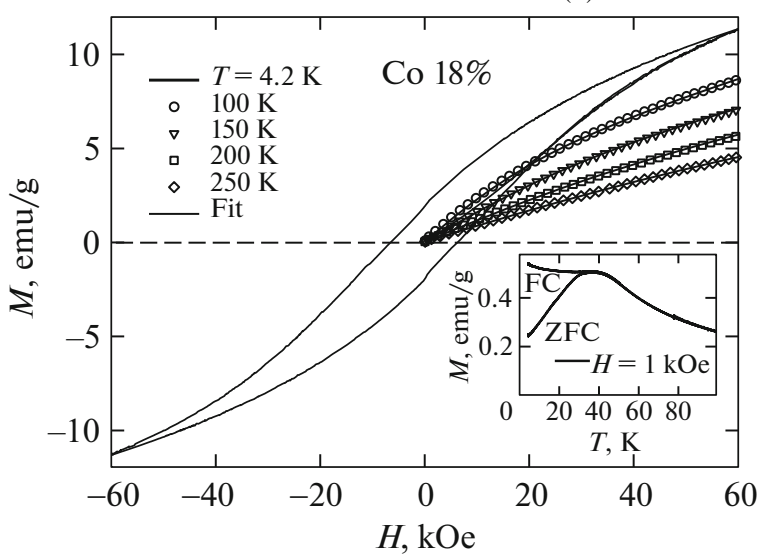

Fig. 5. Magnetization curves at different temperatures for (a) undoped ferrihydrite nanoparticles and (b) ferrihydrite nanoparticles doped with cobalt. Thin solid lines (fit) correspond to the results of the fitting using expression (1). The insets show the temperature dependences of the magnetic moment $M(T)$ measured in the ZFC and FC modes.
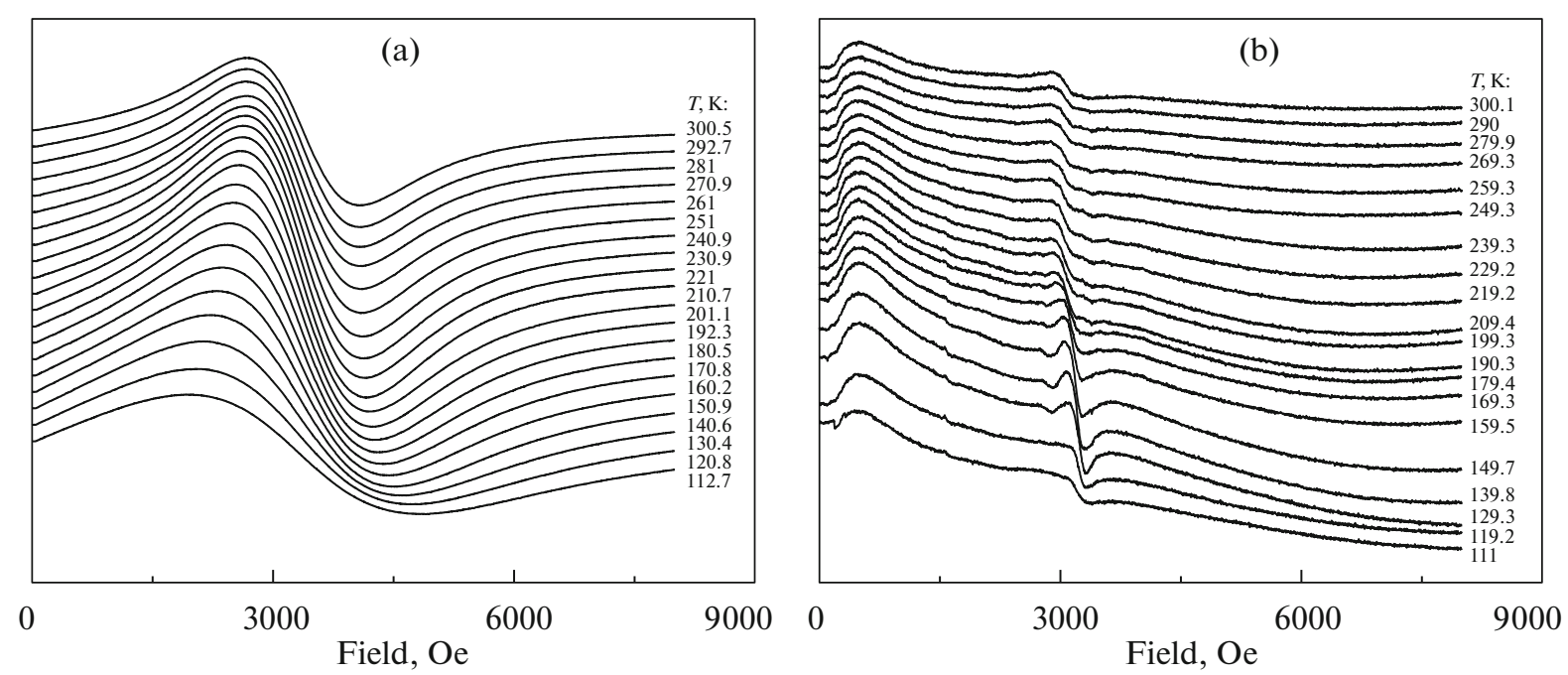

Fig. 6. Ferromagnetic resonance spectra recorded at temperatures in the range from $\sim 100$ to $300 \mathrm{~K}$ for (a) undoped ferrihydrite nanoparticles and (b) ferrihydrite nanoparticles doped with cobalt.

cles are shown in Fig. 6. It can be seen from this figure that the doping of the ferrihydrite nanoparticles with cobalt atoms significantly modifies the absorption spectra. The temperature dependences of the ferromagnetic resonance linewidth $\Delta H(T)$ are shown in Fig. 7. According to the results obtained in [21, 22], for powders of randomly oriented particles of ferromagnets and ferrites, the absorption linewidth is a nonmonotonic function of the temperature: $\Delta H(T)=$ $\Delta H_{s}(T)+\Delta H_{u}(T)$, where $\Delta H_{s}(T)$ is the contribution to the line broadening from the superparamagnetism of nanoparticles, and $\Delta H_{u}(T)$ is the contribution to the

Table 5. Characteristics of the samples prepared

\begin{tabular}{l|c|c|c|c|c}
\hline \multicolumn{1}{c|}{ Sample } & $D, \mathrm{~nm}$ & $T_{B}, \mathrm{~K}$ & $H_{C}, \mathrm{kOe}$ & $M_{S}, \mathrm{G}$ & $\begin{array}{c}\chi_{\mathrm{AF}}, 10^{-4}, \\
\mathrm{cmu} /(\mathrm{g} \mathrm{Oe})\end{array}$ \\
\hline Biological ferrihydrite & 2 & 23.3 & 3.6 & 26 & 0.6 \\
Chemically synthesized ferrihydrite & 2.5 & 40 & 4 & 25 & 7.1 \\
Ferrihydrite doped with cobalt (18 at \%) & 3.5 & 36 & 5.3 & 7 \\
\hline
\end{tabular}



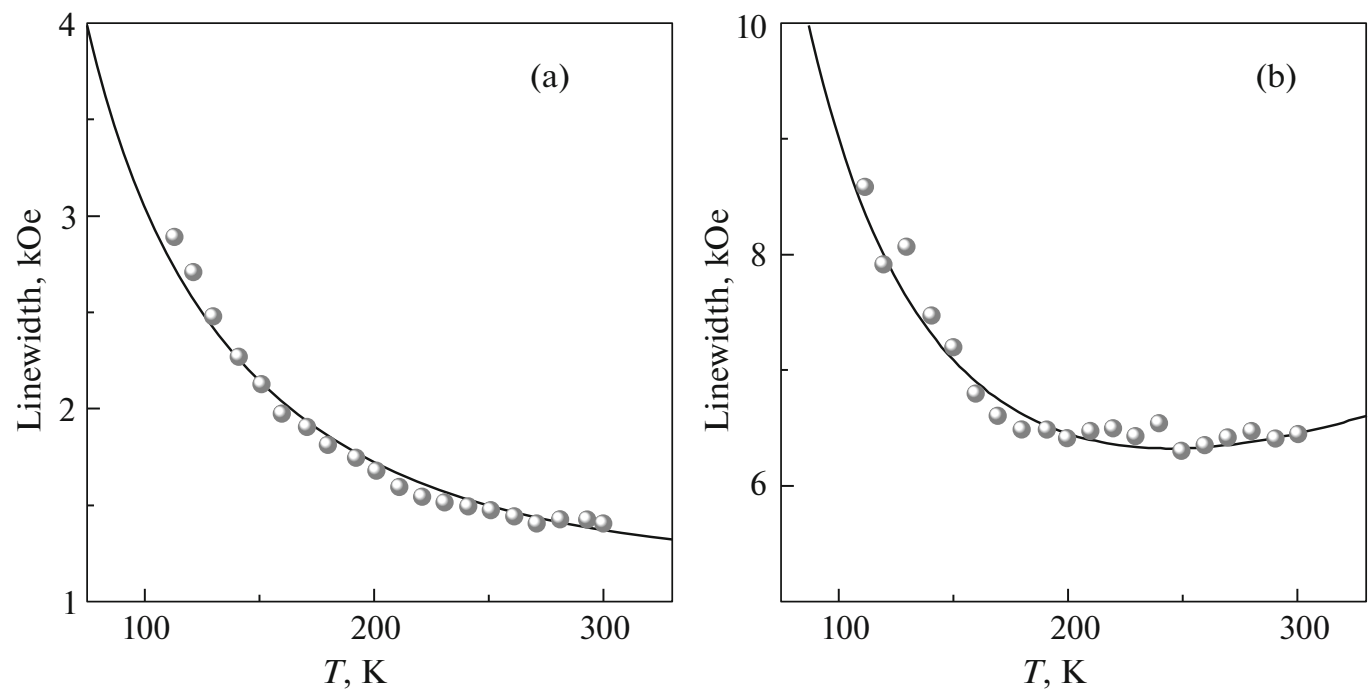

Fig. 7. Temperature dependences of the ferromagnetic resonance linewidth for (a) undoped ferrihydrite nanoparticles and (b) ferrihydrite nanoparticles doped with cobalt. The solid lines show the results of the fitting.

line broadening due to the spread in the directions of the anisotropy fields of nanoparticles (inhomogeneous broadening), which is a decisive factor at low temperatures. The dependences $\Delta H_{s}(T)$ and $\Delta H_{u}(T)$ are the functions of the Langevin parameter $x=$ $(M V \omega) /(\gamma k T)$, where $M$ is the magnetization, $V$ is the volume of the particle, $k$ is the Boltzmann constant, $T$ is the temperature, $\omega$ is the frequency, and $\gamma$ is the gyromagnetic ratio. These functions are determined as follows: $\Delta H_{s}(T)=\omega \alpha\left(x-L_{1}\right) /\left(\sqrt{3} x \gamma L_{1}\right)$ and $\Delta H_{u}(T)=$ $\left(3 \omega \varepsilon L_{2}\right) /\left(\gamma L_{1}\right)$, where $\alpha=0.01$ is the damping parameter, $\varepsilon=K \gamma / M \omega, K$ is the anisotropy constant, and $L_{1,2}$ are the Langevin functions.

The curves shown in Fig. 7 are characterized by two fitting parameters: $K V$ and $M V$. For the case of the initial ferrihydrite nanoparticles, these parameters have the values of $K V=2 \times 10^{-14} \mathrm{erg}$ and $M V=2.4 \times$ $10^{-18} \mathrm{emu}$. For the ferrihydrite nanoparticles doped with cobalt, $K V=5.3 \times 10^{-14} \mathrm{erg}$ and $M V=2.46 \times$ $10^{-19}$ emu. Table 6 presents the obtained values of these fitting parameters, as well as the parameters $K V$ and $M V$ for ferrihydrite nanoparticles of biogenic origin, for which the resonance curves were reported in [20]. This table also presents the corresponding parameters for ferritin complexes, which were calcu- lated from the temperature dependences of the ferromagnetic resonance [23].

For the undoped ferrihydrite nanoparticles, as well as for the ferrihydrite nanoparticles doped with cobalt, in the temperature range under investigation, the intensity of the ferromagnetic resonance signal from the ferrihydrite nanoparticles almost linearly decreased throughout the measured temperature range. This fact indicates that the ferrihydrite nanoparticles are in an unblocked superparamagnetic state.

The temperature dependences of the resonance fields of the ferrihydrite nanoparticles under investigation are shown in Fig. 8. Points of curve 1 in Fig. 8 describe the dependence $H_{r}(T)$ for the undoped ferrihydrite nanoparticles, whereas points of curve 2 in this figure represent the dependence $H_{r}(T)$ for the ferrihydrite nanoparticles doped with cobalt. The resonance field of the undoped ferrihydrite nanoparticles in the temperature range under consideration remains unchanged: $H_{r}=\omega / \gamma=3350$ Oe. For the ferrihydrite nanoparticles doped with cobalt, the resonance field $H_{r}(T)$ increases nonmonotonically with an increase in the temperature $\left(H_{r}=1000 \mathrm{Oe}\right.$ at $T=100 \mathrm{~K}$ and $H_{r}=$ $1600 \mathrm{Oe}$ at $T=300 \mathrm{~K}$ ). In this case, the resonance

Table 6. Fitting parameters obtained for the curves within the framework of the theory [21, 22]

\begin{tabular}{l|c|c|c|c}
\hline \multirow{2}{*}{ Parameter } & \multicolumn{3}{|c|}{ Ferrihydrite } & \multirow{2}{*}{ Ferritin [23] } \\
\cline { 2 - 4 } & biogenic & chemically synthesized & $\begin{array}{c}\text { chemically synthesized }+ \\
\text { cobalt-doped }\end{array}$ & $2.5 \times 10^{-14}$ \\
\hline$K V$, erg & $1.25 \times 10^{-14}$ & $2 \times 10^{-14}$ & $5.3 \times 10^{-14}$ & $1.9 \times 10^{-17}$ \\
\hline
\end{tabular}




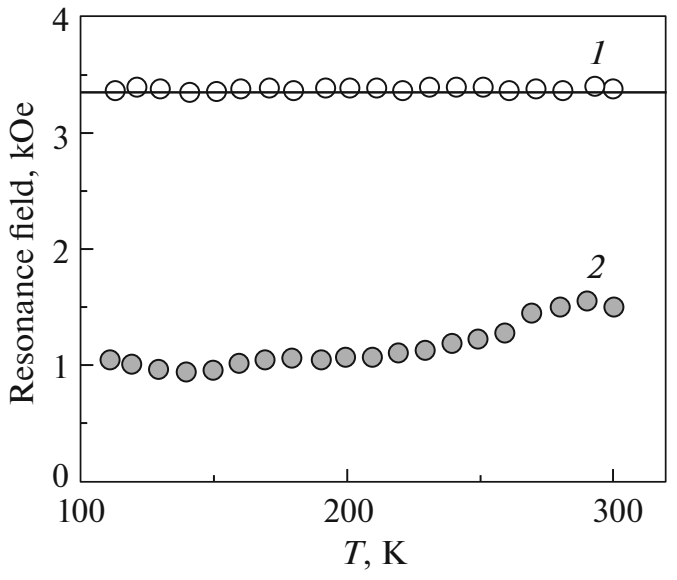

Fig. 8. Dependences of the resonance field on the temperature for (1) undoped ferrihydrite nanoparticles and (2) ferrihydrite nanoparticles doped with cobalt.

field satisfies the relationship $H_{r}<\omega / \gamma$. In other words, there is an isotropic shift of the resonance field $H_{r}$, which depends on the temperature. This shift indicates that, in the ferrihydrite nanoparticles doped with cobalt, there is a surface anisotropy of the rotational type [24]. As is known, the surface magnetic anisotropy is associated with the difference between the symmetry of the environment of the surface atomic magnetic moments and the corresponding symmetry for the magnetic moments of atoms in the bulk of the material.

A type of surface anisotropy is unidirectional exchange anisotropy formed at the ferro(ferri)magnetic-antiferromagnetic interface $[25,26]$. The phenomenon of unidirectional anisotropy, which consists in the existence of a single energetically favorable direction for the magnetization vector $M$, externally manifests itself in the displacement of the hysteresis loop $M(H)$ with respect to the zero value of the applied magnetic field $H$. However, if the crystallographic anisotropy of the antiferromagnetic material is small, no displacement of the hysteresis loop is observed (the coercive field increases), and the magnetic structure of the antiferromagnetic layer will be rotated following the applied external magnetic field. In this case, the surface anisotropy will be the rotational anisotropy. The observation of the unidirectional anisotropy in antiferromagnetic nanoparticles, for example, $\mathrm{NiO}[6$, $27,28]$, as well as in the antiferromagnetic ferrihydrite nanoparticles studied in our work, can be associated with the formation of a "ferromagnetic" surface due to the presence of defects in the material or with the spinglass behavior of the surface also caused by the presence of defects. According to the results obtained in [29, 30], we can write the following expression: $3 K_{u} / M R=\omega / \gamma-H R$, where $K_{u}$ is the surface anisotropy constant, $M$ is the magnetization, and $R$ is the radius of the particle. Substituting into this expression the values of $M$ and $R$ found by fitting the dependences $\Delta H(T)$, we obtain the rotational anisotropy constant $K_{u}=1.6 \times 10^{-3} \mathrm{erg} / \mathrm{cm}^{2}$.

\section{CONCLUSIONS}

Powders of undoped ferrihydrite nanoparticles and ferrihydrite nanoparticles doped with cobalt were prepared by hydrolysis of iron salts. The sizes of nanoparticles and their magnetization, surface and bulk anisotropy constants, and blocking temperatures were determined. The doping of nanoparticles with cobalt leads to a significant increase in the anisotropy constant of a nanoparticle and to the formation of surface rotational anisotropy with the surface anisotropy constant $K_{u}=1.6 \times 10^{-3} \mathrm{erg} / \mathrm{cm}^{2}$.

\section{ACKNOWLEDGMENTS}

This study was supported by the Ministry of Education and Science of the Russian Federation within the framework of the Special Program for the Siberian Federal University, the Russian Foundation for Basic Research (RFBR project no. 16-03-00969), and jointly by the Russian Foundation for Basic Research and the Krasnoyarsk Regional Science Foundation (RFBR-KRSF r-sibir'-a project no. 15-42-04171).

\section{REFERENCES}

1. A.-H. Lu, E. L. Salabas, and F. Schüth, Angew. Chem., Int. Ed. 46, 1222 (2007).

2. E. Murad and U. Schwertmann, Am. Mineral. 65, 1044 (1980).

3. E. Jansen, A. Kyek, W. Schafer, and U. Schwertmann, Appl. Phys. A: Mater. Sci. Process. 74, s1004 (2002).

4. S. V. Stolyar, O. A. Bayukov, Y. L. Gurevich, V. P. Ladygina, R. S. Iskhakov, and P. P. Pustoshilov, Inorg. Mater. 43 (6), 638 (2007).

5. Z. Wang, C. Li, M. Ellenburg, E. Soistman, J. Ruble, B. Wright, J. X. Ho, and D. C. Carter, Acta Crystallogr., Sect. D: Biol. Crystallogr. 62, 800 (2006).

6. S. A. Makhlouf, F. T. Parker, F. E. Spada, and A. E. Berkowitz, J. Appl. Phys. 81 (8), 5561 (1997).

7. J. T. Richardson, D. I. Yiagas, B. Turk, K. Forster, and M. V. Twigg, J. Appl. Phys. 70 (11), 6977 (1991).

8. R. D. Zysler, D. Fiorani, A. M. Testa, L. Suber, E. Agostinelli, and M. Godinho, Phys. Rev. B: Condens. Matter 68 (21), 212408 (2003).

9. X. G. Zheng, C. N. Xu, K. Nishikubo, K. Nishiyama, W. Higemoto, W. J. Moon, E. Tanaka, and E. S. Otabe, Phys. Rev. B: Condens. Matter 72 (1), 014464 (2005).

10. K. Dobretsov, S. Stolyar, and A. Lopatin, Acta Otorhinolaryngologica Ital. 35, 97 (2015).

11. E. V. Inzhevatkin, E. V. Morozov, E. D. Khilazheva, V. P. Ladygina, S. V. Stolyar, and O. V. Falaleev, Bull. Exp. Biol. Med. 158, 807 (2015). 
12. A. Punnoose, T. Phanthavady, M. S. Seehra, N. Shah, and G. P. Huffman, Phys. Rev. B: Condens. Matter 69 (5), 054425 (2004).

13. D. A. Balaev, Yu. V. Boyarshinov, M. M. Karpenko, and B. P. Khrustalev, Prib. Tekh. Eksp., No. 3, 167 (1985).

14. R. K. Kukkadapu, J. M. Zachara, J. K. Fredrickson, S. C. Smith, A. C. Dohnalkova, and C. K. Russell, Am. Mineral. 88, 1903 (2003).

15. Y. Guyodo, S. K. Banerjee, R. Lee Penn, D. Burleson, T. S. Berquo, T. Seda, and P. Solheid, Phys. Earth Planet. Inter. 154, 222 (2006).

16. D. A. Balaev, A. A. Krasikov, A. A. Dubrovskiy, S. I. Popkov, S. V. Stolyar, O. A. Bayukov, R. S. Iskhakov, V. P. Ladygina, and R. N. Yaroslavtsev, J. Magn. Magn. Mater. 410, 171 (2016).

17. N. J. O. Silva, V. S. Amaral, and L. D. Carlos, Phys. Rev. B: Condens. Matter 71 (18), 184408 (2005).

18. C. Gilles, P. Bonville, H. Rakoto, J. M. Broto, K. K. W. Wong, and S. Mann, J. Magn. Magn. Mater. 241, 430 (2002).

19. D. A. Balaev, A. A. Krasikov, A. A. Dubrovskii, S. V. Semenov, O. A. Bayukov, S. V. Stolyar, R. S. Iskhakov, V. P. Ladygina, and L. A. Ishchenko, J. Exp. Theor. Phys. 119 (3), 479 (2014).

20. D. A. Balaev, A. A. Krasikov, S. V. Stolyar, R. S. Iskhakov, V. P. Ladygina, R. N. Yaroslavtsev, O. A. Bayukov,
A. M. Vorotynov, M. N. Volochaev, and A. A. Dubrovskiy, Phys. Solid State 58 (9), 1782 (2016).

21. Yu. L. Raikher and V. I. Stepanov, Sov. Phys. JETP 75 (4), 764 (1992).

22. I. S. Poperechny and Y. L. Raikher, Phys.Rev. B 93 (1), 014441 (2016).

23. E. Wajnberg, L. J. El-Jaick, M. P. Linhares, and D. M. S. Esquivel, J. Magn. Reson. 153, 69 (2001).

24. R. J. Prosen, J. O. Holmen, and B. E. Gran, J. Appl. Phys. 32 (3), S91 (1961).

25. J. Nogués and I. K. Schuller, J. Magn. Magn. Mater. 192, 203 (1999).

26. W. H. Meiklejohn and C. P. Bean, Phys. Rev. 102 (5), 1413 (1956).

27. S. A. Makhlouf, H. Al-Attar, and R. H. Kodama, Solid State Commun. 145, 1 (2008).

28. H. Bi, S. Li, Y. Zhang, and Y. Du, J. Magn. Magn. Mater. 277, 363 (2004).

29. F. Gazeau, J. C. Bacri, F. Gendron, R. Perzynski, Yu. L. Raikher, V. I. Stepanov, and E. Dubois, J. Magn. Magn. Mater. 186, 175 (1998).

30. F. Gazeau, V. Shilov, J. C. Bacri, E. Dubois, F. Gendron, R. Perzynski, Y. L. Raikher, and V. I. Stepanov, J. Magn. Magn. Mater. 202, 535 (1999).

Translated by O. Borovik-Romanova 